\title{
Fast Unsupervised Texture Segmentation Using Active Contours Model Driven by Bhattacharyya Gradient Flow
}

\author{
Foued Derraz ${ }^{1,2}$, Abdelmalik Taleb-Ahmed ${ }^{1}$, Antonio Pinti ${ }^{1}$, Laurent Peyrodie ${ }^{4}$, \\ Nacim Betrouni ${ }^{3}$, Azzeddine Chikh $^{2}$, and Fethi Bereksi-Reguig ${ }^{2}$ \\ ${ }^{1}$ LAMIH UMR CNRS 8530, Le Mont Houy, 59313 Valenciennes, France \\ ${ }^{2}$ GBM Laboratory Abou Bekr Belkaid university Tlemcen, 13000, Algeria \\ ${ }^{3}$ LAMIH UMR CNRS 8530, Le Mont Houy, 59313 Valenciennes \\ ${ }^{4}$ Hautes Etudes d'Ingénieur Lille, France \\ f_derraz@hotmail.com, taleb@univ-valenciennes.fr, \\ Antonio.pinti@univ-valenciennes.fr, laurent.peyrodie@hei.fr, \\ n_betrouni@chu-lille.fr, az_chikh@hotmail.com, \\ f_bereksi@mail.univ-tlemcen.dz
}

\begin{abstract}
We present a new unsupervised segmentation based active contours model and texture descriptor. The proposed texture descriptor intrinsically describes the geometry of textural regions using the shape operator defined in Beltrami framework. We use Bhattacharyya distance to discriminate textures by maximizing distance between the probability density functions which leads to distinguish textural objects of interest and background. We propose a fast Bregman split implementation of our segmentation algorithm based on the dual formulation of the Total Variation norm. Finally, we show results on some challenging images to illustrate segmentations that are possible.
\end{abstract}

Keywords: Active contours, texture descriptor, bhattacharyya distance, total variation, Bregman split algorithm.

\section{Introduction}

Active contour models such as Geometric/Geodesic Active Contour (GAC) [1], Active Contours Without Edge (ACWE) [7] model have been widely used as image segmentation methods and more recently for texture segmentation [4]. Later, extension and generalization [8],[13],[16],[18]-[22], respectively, for vector-valued images has been done by replacing the scalar gray-level intensities with vectors of color channel intensities to guide contour evolution. However, the information derived from intensity integral operations deceived the textural image segmentation process as regions of different textures may have equal average intensities. Therefore the application ACWE model based on image intensities either in its original or in its generalized form can be considered unsuitable for texture segmentation. However, its regionbased formulation could be exploited for capturing textural information derived from features not necessarily exhibiting high gradients at object boundaries. 
In this paper, we proposed fast unsupervised segmentation algorithm to textural segmentation model based on Bhattacharyya distance.

The rest of paper is organized as follows. Firstly, we introduce the texture region and shape descriptor. Then, we define the active contour model based on the Bhattacharyya distance. We prove the existence of a minimizer. We then present the fast algorithm to determine evolving contour curve. Finally, we show some promising experimental results.

\section{Texture Descriptor}

In Beltrami framework [2], a smoothed version of an original gray level image $I: \mathbb{R}^{2} \rightarrow \mathbb{R}^{+}$can be viewed as a surface $\sum$ with local coordinates $(x, y)$ embedded in $\mathbb{R}^{3}$ by smooth mapping $X$. Let $(x, y) \rightarrow X=\left(x, y, G_{\sigma} * I\right), G_{\sigma}$ is Gaussian filter with $\sigma^{2}$ variance, the first fundamental form is define by:

$$
g_{\mu, v}(x, y)=\left(\left\langle\frac{\partial X}{\partial \mu}, \frac{\partial X}{\partial v}\right\rangle\right)=\left(\begin{array}{cc}
1+\hat{I}_{x}^{2} & \hat{I}_{x} \hat{I}_{y} \\
\hat{I}_{x} \hat{I}_{y} & 1+\hat{I}_{y}^{2}
\end{array}\right)
$$

where $\mu, v=x, y$ in the $(x, y)$-basis and $\hat{I}_{x}$ and $\hat{I}_{y}$ in (1) are the image derivatives convolved with a relatively large Gaussian filter, such $\hat{I}_{x}=G_{\sigma} * I_{x}$ and $\hat{I}_{y}=G_{\sigma} * I_{y}$.

The inverse determinant (det) of metric tensor $g_{\mu, \nu}$ is defined as:

$$
g(I)=\frac{1}{\operatorname{det}\left(g_{\mu, v}(x, y)\right)}=\frac{1}{1+\left|\nabla G_{\sigma} * I\right|^{2}}
$$

Instead of this edge descriptor [3], we propose to define a region descriptor for textural image. For this, we design an intrinsic descriptor based on the use of shape operator to describe the geometry of textures [8]. The shape operator $S$ measures the shape of the manifold in a given region by estimating how the normal $N_{\Sigma}$ to the surface $\Sigma$ changes from point to point. For a tangent vector $v_{p}$ to $\Sigma$ at $p$, the shape operator that satisfying [8]:

$$
S=-D_{v_{p}} N_{\Sigma}
$$

where $D_{v_{p}} N_{\Sigma}$ is the derivative of the surface normal in the direction $v_{p}$.

The second fundamental form $b_{\mu, v}$, used to measure oriented distance on manifolds, and its components indicate the direction of change of the manifold as: 


$$
b_{\mu, \nu}(x, y)=\left(\left\langle N_{\Sigma}, \frac{\partial^{2} X}{\partial \mu \partial \nu}\right\rangle\right)=\frac{1}{\sqrt{1+\hat{I}_{x}^{2}+\hat{I}_{y}^{2}}}\left(\begin{array}{ll}
\hat{I}_{x x} & \hat{I}_{x y} \\
\hat{I}_{x y} & \hat{I}_{y y}
\end{array}\right)
$$

where $N_{\Sigma}=\frac{1}{\sqrt{1+\hat{I}_{x}^{2}+\hat{I}_{y}^{2}}}\left(-\hat{I}_{x},-\hat{I}_{y}, 1\right)$ is normal to surface manifold.

The shape operator $S$ calculates the bending of a smoothed surface in different directions [8]. The principles curvatures of the manifold are the roots of the following equation:

$$
k^{2}-b_{\mu v}(x, y) g^{\mu v}(x, y) k+\frac{b}{g}=0
$$

where $g^{\mu v}(x, y)=g_{\mu v}{ }^{-1}(x, y), b=\operatorname{det}\left(b_{\mu, v}(x, y)\right)$ and $g=\operatorname{det}\left(g_{\mu, v}(x, y)\right)$.

The first principal curvature $\kappa_{\max }$ corresponds to the maximal change of the normal to the surface and $\kappa_{\min }$ corresponds to the minimum change:

$$
\begin{aligned}
& \kappa_{\max }=-\frac{1}{2} \operatorname{trace}\left(b_{\mu, v}(x, y) g^{\mu, v}(x, y)\right)+\sqrt{\frac{1}{4}\left(\operatorname{trace}\left(b_{\mu, v}(x, y) g^{\mu, v}(x, y)\right)\right)^{2}-\frac{b}{g}} \\
& \kappa_{\min }=-\frac{1}{2} \operatorname{trace}\left(b_{\mu, v}(x, y) g^{\mu, v}(x, y)\right)-\sqrt{\frac{1}{4}\left(\operatorname{trace}\left(b_{\mu, v}(x, y) g^{\mu, v}(x, y)\right)\right)^{2}-\frac{b}{g}}
\end{aligned}
$$

where $b=\frac{1}{Z}\left(\hat{I}_{x x} \hat{I}_{y y}-\hat{I}_{x y}^{2}\right), g=Z^{2}, Z=\sqrt{1+\hat{I}_{x}^{2}+\hat{I}_{y}^{2}}$

and $b_{\mu, v}(x, y) g^{\mu, v}(x, y)=\frac{1}{g Z}\left(\begin{array}{ll}\hat{I}_{x x}\left(1+\hat{I}_{y}^{2}\right)+\hat{I}_{x y} \hat{I}_{x} \hat{I}_{y} & \hat{I}_{x y}\left(1+\hat{I}_{x}^{2}\right)+\hat{I}_{x x} \hat{I}_{x} \hat{I}_{y} \\ \hat{I}_{x y}\left(1+\hat{I}_{y}^{2}\right)+\hat{I}_{y y} \hat{I}_{x} \hat{I}_{y} & \hat{I}_{y y}\left(1+\hat{I}_{x}^{2}\right)+\hat{I}_{x y} \hat{I}_{x} \hat{I}_{y}\end{array}\right)$,

Since $\kappa_{\max } \perp \kappa_{\min }$, we propose to define the texture descriptor as:

$$
\kappa_{T}=\operatorname{atan}\left(\frac{\kappa_{\max }}{\kappa_{\min }}\right)
$$

Where $\kappa_{T}: \Omega \rightarrow \mathbb{R}^{+}$is used to segment regions with different texture patterns, $\Omega$ corresponds to the image domain. 


\section{Bhattacharyya Flow}

The Bhattacharyya distance between two probability density functions is defined as $E_{B a t}=-\log (B a t)$, where Bat is the Bhattacharyya coefficient given by [16,17]:

$$
\operatorname{Bat}\left(p_{\text {in }}, p_{\text {out }}\right)=\int_{R^{+}} \sqrt{p_{\text {in }}\left(\kappa_{T}, \Omega\right) p_{\text {out }}\left(\kappa_{T}, \Omega\right)} d \kappa_{T}
$$

The pdfs $p_{\text {in }}$ and $p_{\text {out }}$ associated with an observation $\kappa_{T}$ for a fixed region $\Omega$ are defined by the Parzen kernel:

$$
\left\{\begin{array}{c}
p_{\text {in }}\left(\kappa_{T}, \Omega\right)=\frac{1}{|\Omega|} \int_{\Omega} K_{\sigma_{\mathrm{ker}}}\left(\kappa_{T}-\kappa_{T}(x)\right) d x \\
p_{\text {out }}\left(\kappa_{T}, \Omega\right)=\frac{1}{\left|\Omega_{0} \backslash \Omega\right|} \int_{\Omega_{0} \backslash \Omega} K_{\sigma_{\mathrm{ker}}}\left(\kappa_{T}-\kappa_{T}(x)\right) d x
\end{array}\right.
$$

In order to produce two regions, the object $\Omega$ and the background $\Omega_{0} \backslash \Omega$, with two pdfs as disjoint as possible, the energy the functional $E_{B a t}$ is maximized, w.r.t the evolving domain $\Omega(t)$, is done with the shape derivative tool[4,10]. Thus, the Eulerian derivative of $E_{B a t}$ in the direction $\xi$ is as follows:

$$
\left\langle\frac{\partial E_{B a t}(\Omega(t))}{\partial t}, \xi\right\rangle=\int_{\partial \Omega} V_{B a t}\left\langle\xi(s), N_{C}(s)\right\rangle d s
$$

where the Bhattacharyya velocity is expressed as:

$$
\begin{aligned}
V_{\text {Bat }} & =\frac{1}{2}\left(\frac{1}{|\Omega|}-\frac{1}{\left|\Omega_{0} \backslash \Omega\right|}\right) \sqrt{P_{\text {in }}\left(\kappa_{T}, \Omega\right) P_{\text {out }}\left(\kappa_{T}, \Omega\right)} \\
& +\frac{1}{2\left|\Omega_{0} \backslash \Omega\right|} \int_{R^{+}} \sqrt{\frac{P_{\text {in }}\left(\kappa_{T}, \Omega\right)}{P_{\text {out }}\left(\kappa_{T}, \Omega\right)}\left(\begin{array}{l}
K_{\sigma_{\mathrm{krr}}}\left(\kappa_{T}-\kappa(s)\right) \\
-\sqrt{P_{\text {out }}\left(\kappa_{T}, \Omega\right)}
\end{array}\right) d \kappa_{T}} \\
& -\frac{1}{2|\Omega|} \int_{R^{+}} \sqrt{\frac{P_{\text {out }}\left(\kappa_{T}, \Omega\right)}{P_{\text {in }}\left(\kappa_{T}, \Omega\right)}}\left(\begin{array}{l}
K_{\sigma_{\mathrm{ker}}}\left(\kappa_{T}-\kappa(s)\right) \\
-\sqrt{P_{\text {in }}\left(\kappa_{T}, \Omega\right)}
\end{array}\right) d \kappa_{T}
\end{aligned}
$$

Where $\vec{N}$ is an exterior unit normal vector to the boundary $C=\partial \Omega$ of the region $\Omega$, $\langle\varepsilon, \vec{N}\rangle$ is the Euclidean scalar product and $s$ is the arc length parametrization. If we consider the energy functional expressed as:

$$
E(\Omega)=L_{g}(\Omega)+\lambda E_{B a t}(\Omega)
$$

where $L_{g}(\Omega)$ is the length of the boundary of $\Omega$ and acts like a regularization process in the curve evolution, $\lambda$ positive constant which controls the trade-off between the 
regularization process and the fidelity of the solution. In Total variation norm the energy of active contours can be expressed as:

$$
\min _{u \in[0,1]}(E(u))=\lambda \int_{\Omega_{0}} V_{B a t} u+\int_{\Omega_{0}} g(I)|\nabla u|
$$

In the next section we introduced a fast algorithm for solving segmentation problem.

\section{Fast Algorithm Based on Split Bregman}

A fast and accurate minimization algorithm for (16) is introduced in [5]. We substitute $\phi$ by $u$ to formulate the variational problem:

$$
\min _{u \in[0,1]}(E(u))=\lambda \int_{\Omega_{0}} V_{B a t} u+\int_{\Omega_{0}} g(I)|\nabla u|
$$

A new vectorial function $d$ is introduced as follows:

$$
\min _{u \in[0,1], d}\left(\lambda \int_{\Omega_{0}} V_{B a t} u+\int_{\Omega_{0}} g(I)|d|\right)
$$

The constraintis $d=\nabla u$ enforced using the efficient Bregman iteration approach [10, 13, 3] defined as:

$$
\left\{\begin{array}{c}
\left(u^{k+1}, d^{k+1}\right)=\arg \min \left(\left\{\lambda \int_{\Omega_{0}} V_{B a t} u+\int_{\Omega_{0}} g(I)|d|+\frac{\mu}{2} \int_{\Omega_{0}}\left|d-\nabla u-b^{k}\right|^{2}\right\}\right) \\
b^{k+1}=b^{k}+\nabla u^{k}-d^{k+1}
\end{array}\right.
$$

The minimizing solution $u^{k+1}$ is characterized by the optimality condition:

$$
\mu \Delta u=\lambda V_{b a t}+\mu \operatorname{div}\left(b^{k}-d^{k}\right), u \in[0,1]
$$

A fast approximated solution is provided by a Gauss-Seidel iterative scheme:

$$
\left\{\begin{array}{c}
\gamma_{i, j}=d_{i-1, j}^{x, k}-d_{i, j}^{x, k}-b_{i-1, j}^{x, k}+b_{i, j}^{x, k}+d_{i, j-1}^{y, k}-d_{i, j}^{y, k}-b_{i, j-1}^{y, k}+b_{i, j}^{y, k} \\
\mu_{i, j}=\frac{1}{4}\left(u_{i-1, j}^{k, n}+u_{i+1, j}^{k, n}+u_{i, j+1}^{k, n}+u_{i, j+1}^{k, n}-\frac{\lambda}{\mu} V_{B a t, i, j}+\gamma_{i, j}\right), n>0, k>0 \\
u_{i, j}^{k+1, n+1}=\max \left\{\min \left\{\mu_{i, j}, 1\right\}, 0\right\}
\end{array}\right.
$$

Finally, the minimizing solution $d^{k+1}$ is given by soft-thresholding: 


$$
d_{i, j}^{k+1}=\frac{\nabla u^{k+1}+b^{k}}{\left|\nabla u^{k+1}+b^{k}\right|} \max \left(\left|\nabla u^{k+1}+b^{k}\right|-\mu^{-1}, 0\right)
$$

Then, the final active contour is given by the boundary of the set $\left\{\mathbf{x} \in \Omega \mid u^{\text {final }}>\frac{1}{2}\right\}$. The two iteration schemes are straightforward to implement. Finally, we update at each iteration $p_{\text {in }}, p_{\text {out }}$ using the Parzen kernel given in equation (10).

\section{Experiments Results}

We applied the proposed segmentation algorithm to a set of challenging real-world textural images (image a),b),c))). The natural textural images were taken in the Berkeley segmentation data set 14. Fig. 1 presents the results obtained with the proposed method. We notice that our segmentation model needs four parameters, $\sigma$ explained in section 2. $\theta, \lambda$ explained in Section 4. The Parzen parameter in Section 3. The mean computing time for the segmentation is around a minute. The segmentation results are compared to manual segmentation [14], and we evaluation the quality of segmentation in term of F-measure detailed in [14]. For a good choice of the segmentation parameters, the results are compared to manual segmentation and the Fmeasure drawn (Table 1)an improvement of segmentation quality compared to results drawn by the model proposed in [3].

Table 1. Quatitative evaluation of the segmentation

\begin{tabular}{|c|c|c|c|}
\hline Image & $\mathbf{P}$ & $\mathbf{R}$ & $\mathbf{F}$ \\
\hline Image a & 0.61 & 0.59 & 0.60 \\
\hline Image b & 0.63 & 0.60 & 0.61 \\
\hline Image c & 0.65 & 0.62 & 0.63 \\
\hline
\end{tabular}

Integrating the texture region descriptor guide the active contour to localize efficiently the geometry of textured region. Solving the segmentation problem in dual TV allows the active contour to reach the minimum global and ensure the active contour to segment the one textural region in image. We have compared the segmentation results of image a), b), c) to the manual segmentation. The quality of segmentation expressed in F-measure term shows that the proposed method segments successfully the textured regions. An adequate choice of parameters model leads to a good segmentation of textural image. 

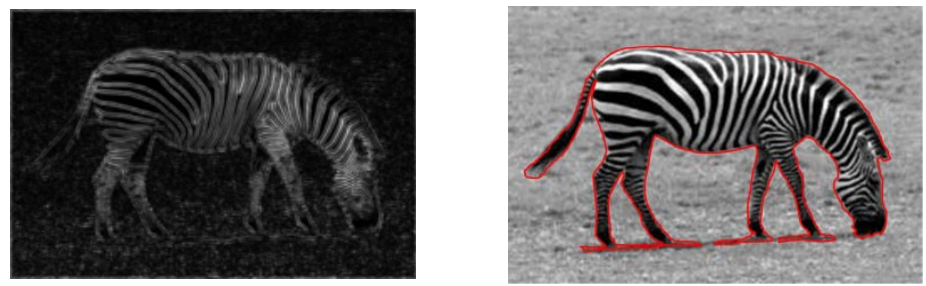

a)
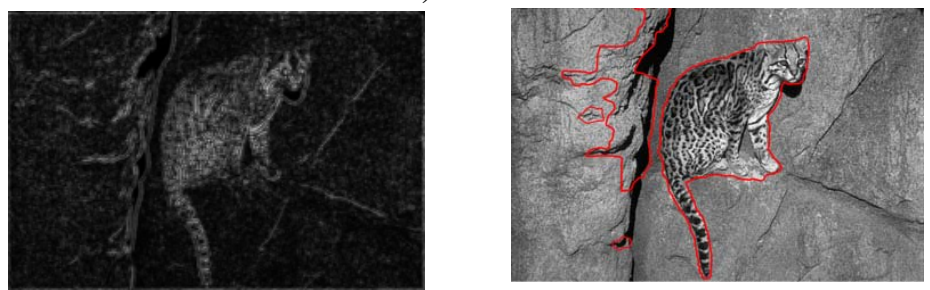

b)
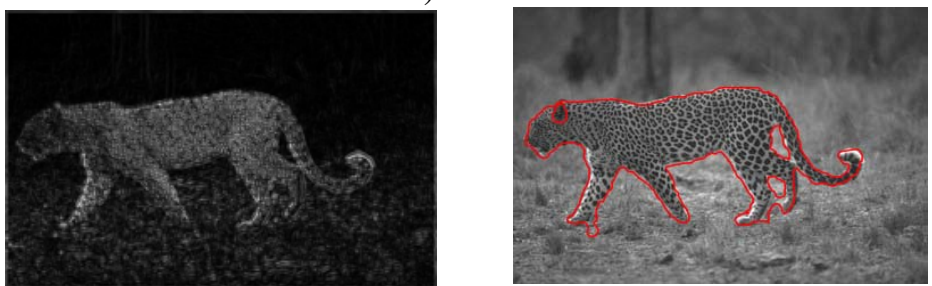

c)

Shape descriptor

Segmentation results

Fig. 1. Segmentation of textural images based on bhattacharyya distance

\section{Conclusion}

We have introduced an active contour model based Bhattacharyya gradient flow for unsupervised segmentation of textural images. We have proposed a new intrinsic textural feature descriptor based on the shape operator of the texture manifold and fast algorithm is developed based on a dual TV approach. The proposed model is designed to work with textures and needs at least one textural region.

\section{References}

1. Caselles, V., Kimmel, R., Sapiro, G.: Geodesic Active Contours. IJCV 22(1), 61-97 (1997)

2. Sochen, N., Kimmel, R., Malladi, R.: A general framework for low level vision. IEEE TIP 7(3), 310-318 (1996)

3. Sagiv, C., Sochen, N., Zeevi, Y.: Integrated active contours for texture segmentation. IEEE TIP 15(6), 1633-1646 (2006)

4. Aujol, J.-F., Gilboa, G., Chan, T., Osher, S.: Structure-Texture Image DecompositionModeling, Algorithms, and Parameter Selection. IJCV 67(1), 111-136 (2006) 
5. Bresson, X., Esedoglu, S., Vandergheynst, P., Thiran, J., Osher, S.: Fast Global Minimization of the Active Contour/Snake Model. JMIV 28(2), 51-167 (2007)

6. Mi, A.S., Iakovidis, D.K., Maroulis, D.: LBP-guided active contours. Pattern Recognition Letters 29(9), 1404-1415 (2008)

7. Chan, T., Vese, L.: Active Contours Without Edges. IEEE TIP 10(2), 266-277 (2001)

8. Delfour, M., Zolésio, J.: Shapes and Geometries: Analysis, Differential Calculus, and Optimization. Advances in Design and Control, SIAM (2001)

9. Freedman, D., Zhang, T.: Active contours for tracking distributions. IEEE TIP 13(4), 518526 (2004)

10. Herbulot, A., Jehan-Besson, S., Duffiner, S., Barlaud, M., Aubert, G.: Segmentation of vectorial image features using shape gradients and information measures. JMIV 25(3), 365-386 (2006)

11. Rousson, M., Brox, T., Deriche, R.: Active unsupervised texture segmentation on a diffusion based feature space. In: Proc. IEEE CVPR 2003, Madison, WI, USA, vol. 2, pp. 699704 (2003)

12. Lee, S.M., Abott, A.L., Clark, N.A., Araman, P.A.: Active contours on statistical manifolds and texture segmentation. In: Proc. IEEE ICIP 2005, vol. 3, pp. 828-831 (2005)

13. Chan, T., Sandberg, B., Vese, L.: Active contours without edges for vector-valued images. JVCIR 11(2), 130-141 (2000)

14. Martin, D., Fowlkes, C., Malik, J.: Learning to Detect Natural Image Boundaries Using Local Brightness, Color, and Texture Cues. IEEE PAMI 26(5), 530-549 (2004)

15. Goldstein, T., Bresson, X., Osher, S.: Geometric Applications of the Split Bregman Method: Segmentation and Surface Reconstruction. In: Technical Report 06, Math. Department UCLA, Los Angeles, USA (2009)

16. Michailovich, O., Rathi, Y., Tannenbaum, A.: Image Segmentation Using Active Contours Driven by the Bhattacharyya Gradient Flow. IEEE TIP 16(11), 2787-2801 (2007)

17. Raubera, T.W., Braunb, K.B.: Probabilistic distance measures of the Dirichlet and Beta distributions. Pattern Recognition 41(2), 637-645 (2008)

18. Lecellier, F., Fadili, J., Jehan-Besson, S., Aubert, G., Revenu, M.: Region-based active contours and sparse representations for texture segmentation. In: Proc. IEEE ICPR 2008, Florida (2008)

19. Allili, M.S., Ziou, D., Bentabet, L.: A robust level set approach for image segmentation and statistical modeling. In: Proc. Adv. Conc. on Intelligent Vision Systems (ACIVS), pp. 243-251 (2004)

20. Sandberg, B., Chan, T., Vese, L.: A level-set and gabor-based active contour algorithm for segmenting textured images. In: Technical Report 39, Math. Department UCLA, Los Angeles, USA (2002)

21. Yin, W., Osher, S., Goldfarb, D., Darbon, J.: Bregman iterative algoruithms for 11 minimization with applications to compressed sensing. SIAM J. Imaging Sci. 1, 143-168 (2008)

22. He, Y., Luo, Y., Hu, D.: Unsupervised Texture Segmentation via Applying Geodesic Active Regions to Gaborian Feature Space. IEEE Transactions on Engineering, Computing and Technology, 272-275 (2004) 